\title{
Cochlear Implant Failure in the Pediatric Population
}

\author{
Fulya Ozer, Haluk Yavuz, Ismail Yilmaz, and Levent N. Ozluoglu \\ Department of Otorhinolaryngology, Head and Neck Surgery, Baskent University Faculty of Medicine, Ankara, Turkey
}

$\begin{array}{ll}\text { Received } & \text { May 31, 2021 } \\ \text { Revised } & \text { August 16, 2021 } \\ \text { Accepted } & \text { August 23, 2021 }\end{array}$

Address for correspondence

Fulya Ozer, MD, PhD

Department of Otorhinolaryngology,

Head and Neck Surgery,

Baskent University

Faculty of Medicine,

Ankara, Turkey

Tel +90-322-4586868

Fax +90-322-5020011

E-mail fdeveci06@hotmail.com

This study was presented on a round table presentation in the 14th European Symposium of Pediatric Cochlear Implantation, October 16-19, 2019 , Bucharest in Romania.
Background and Objectives: In cochlear implant (Cl) surgery, the results and causes of revision and reimplantation may guide surgeons in establishing surgical protocols for revision surgery with safe audiological outcomes. The aim of this study was to review our experience in terms of etiology, surgical strategy, and hearing outcomes in pediatric patients who underwent $\mathrm{Cl}$ removal and reimplantation. Subjects and Methods: All patients received implants of the same brand. Pre and postoperative Categories of Auditory Performance score and aided free-field pure tone audiometry thresholds were noted. In vivo integrity tests were performed for each patient and the results of ex vivo tests of each implant were obtained from manufacturer. Results: A total of $149 \mathrm{Cls}$ were placed in 121 patients aged $<18$ years. The revision rate in children was $6.7 \%$ (10/121 children). Six patients had a history of head injury leading to a hard failure. The causes of reimplantation in others were soft failure $(n=1)$, electrode migration $(n=1)$, infection $(n=1)$, and other $(n=1)$. All patients showed better or similar postreimplantation audiological performance compared with pre-reimplantation results. Conclusions: It is very important to provide a safe school and home environment and educate the family for reducing reimplantation due to trauma. Especially for active children, psychiatric consultation should be continued postoperatively. J Audiol Otol 2021;25(4):217-223

Keywords: Cochlear implants; Reimplantation; Hard failure; Child.

\section{Introduction}

Due to the increasing prevalence and effectiveness of the newborn hearing screening program, more cochlear implants (CIs) are implanted at an early age in children. Although CI is designed for a lifetime, implantation surgery is not entirely safe and CI carries risk of failure like other implantable devices $[1,2]$.

In the literature, the first CI revision surgery was reported by Hochmair-Desoyer and Burian in 1985 [3]. As the indications of CI widened and the number of surgeries increased, publications on $\mathrm{CI}$ revision surgery increased as well. The rate of revision surgery reported in the literature has ranged from $3.8 \%$ to $7.2 \%$ [4].

The indications of revision of CI surgery were classified by Zeitler, et al. [4]. It includes hard device failure, soft device failure, device infection or extrusion, inadequate initial place-

This is an Open Access article distributed under the terms of the Creative Commons Attribution Non-Commercial License (https://creativecommons.org/licenses/by-nc/4.0/) which permits unrestricted non-commercial use, distribution, and reproduction in any medium, provided the original work is properly cited. ment, surgical wound or flap complications, and improved technology of CI. When the complete interruption of auditory input and a malfunction of communication between the internal-external parts are present, then we can say that there is a hard device failure. It can be proved by in vivo integrity testing $[4,5]$. Soft device failure is suspected with an awareness of declining performance or deterrent symptoms such as a popping or shocking sensation [6]. Also, soft device failure may be considered in patients with non-auditory complaints such as pain, facial nerve stimulation, vertigo, or tinnitus [7]. If the infection persists despite the use of antibiotics, then the device and/or electrode migration and decreased auditory performance can be accompanied. In this situation, the delay time between explantation/reimplantation is recommended $[4,7]$. Electrode extrusion accompanied by a decline in auditory perception is another indication of revision surgery $[4,8]$.

Many previous publications have reported that revision surgeries of CI are seen mostly in children [4,9,10]. An increased rate of head trauma, head growth, and increased rate of otitis media in this population have all been cited as potential causes of increased CI failure and revision surgery $[1,4,9]$. In 
children, timing and deciding of revision CI surgery is very important. Because the disruption in auditory input and language development interruption may have serious consequences such as delay in education and speaking [10].

The majority of reported CI revision and reimplantation surgeries have good results and patients have better outcomes than their best results with their first implant $[1,4,11]$. Reis, et al. [11] compared the speech recognition score (SRS) outcomes before and after CI reimplantation surgery and found that postoperative SRS outcomes were similar or better than preoperative best results. Besides SRS, many methods were used for evaluation of audiological outcomes of reimplantation in children such as Categories of Auditory Performance (CAP), pure tone average (PTA) thresholds, and speech perception in quiet and noise [12-15].

Our CI center is a tertiary center with 600 beds. Our center started the CI program in 2004. Since 2017, we have been performing bilateral $\mathrm{CI}$ on children before 48 months in our country as simultaneously. The aim of this study is a retrospective review of our experience in pediatric patients who underwent CI explantation-reimplantation in terms of etiology, surgical strategy, and hearing outcomes.

\section{Subjects and Methods}

\section{Study design}

This study was approved by Baskent University Ethical and Institutional Review Board (Project no: KA19/310). A retrospective chart review of all pediatric patients under 18 years old undergone the CI surgery between January 2010 and January 2018 were reviewed and from these populations, patients who undergo the revision surgery and explantation with or without reimplantation were detected. "Informed Consent Form for Scientific Research" was taken from all participants for publication of their results.

\section{Data collection}

The following data were collected from the patients' records; demographics, etiology of hearing loss, age at first implantation, the reason for explantation/reimplantation, time from the first implantation to revision, surgical findings, device model and references, pre and postoperative results of category audiological performance scores and free-field PTA thresholds $(0.5,1,2$, and $4 \mathrm{kHz})$.

In vivo integrity tests were performed for each patient and the results of ex vivo tests of each implant were obtained from manufacturer. Then the etiologies of device failure required revision surgery were classified according to Zeitler, et al. [4].

Currently, we use devices of all 4 implant manufacturers.
However before the end of study, we noticed that the largest number of implants were brand of Neurelec/Oticon (Vallauris, France). Therefore, we examined the results of the Neurelec/ Oticon CI brand to prevent effect of the differences between the devices of the companies on our results.

\section{Surgical technique}

All patients were undergone from experienced surgeons' team during both the first implantation and revision surgery. A postauricular small incision is made and mastoidectomy, posterior tympanotomy is done. For positioning of receiver-stimulator, titanium screws are fixed and so that the drilling of bone-bed is not needed. The round window approach is applying from 2009 in our center. A small piece of temporal muscle is used to seal the window after the insertion of the electrode array. Telemetry tests, electrical auditory brainstem responses (e-ABR), and stapedius reflex test are done and reported. All mastoidectomy cavity is filled with Ticcel ${ }^{\mathbb{B}}$ (Eczacıbaşı-Baxter, Istanbul, Turkey).

During reimplantation surgery, the same postauricular incision is repeated with an extended length. Monopolar electrocautery is not used. The newly formed bone around the receiver-stimulator is drilled with a diamond tour. The screws are removed with the elevator because screws cannot be opened with their own screwdriver. The new bone formation and soft tissue and fibrosis around the round window niche were seen and removed gently. Then electrode array pulled elegantly after the preparation of a new implant.

\section{Statistical analysis}

Device failure rate and cumulative survival rate were determined based on European consensus guidelines [5].

Statistical Package for the Social Sciences (SPSS) version 17.0 (SPSS Inc., Chicago, IL, USA) was used to analyze the data. "Paired samples t-test" was applied to compare the results of preoperative and postoperative audiological test. $\mathrm{Cu}$ mulative survival rate curve was used to compare the interval of implantation to reimplantation. Significance was accepted as $p<0.05$ for all tests.

\section{Results}

Between 2010 and 2018, a total of 210 primary CIs of Neurelec/Oticon were performed in 182 patients (121 children, 61 adults). A total of 149 CIs were used in 121 patients under 18 years old. Ten pediatric patients and one adult underwent revision surgery. All patients were revised by the same manufacture device. When the 11 revision procedures were evaluated, an overall institution-specific revision rate was 5.2\%. 
Table 1. Characteristics and audiological outcomes of the patients

\begin{tabular}{|c|c|c|c|c|c|c|c|}
\hline \multirow{2}{*}{ Patient } & \multirow{2}{*}{$\begin{array}{l}\text { Time Interval between } \\
\text { implant/reimplant (mo) }\end{array}$} & \multirow{2}{*}{$\begin{array}{l}\text { Revison } \\
\text { reason }\end{array}$} & \multirow{2}{*}{$\begin{array}{c}\text { First implantation } \\
\text { age }(y r)\end{array}$} & \multicolumn{2}{|c|}{ Pre-reimplant } & \multicolumn{2}{|c|}{ Post-reimplant } \\
\hline & & & & PTA (dBHL) & CAP score & PTA (dBHL) & CAP score \\
\hline 1 & 71 & Device & $>4$ & 35 & 6 & 35 & 5 \\
\hline 2 & 1 & Device & $>4$ & 26 & 6 & 22.8 & 7 \\
\hline 3 & 48 & Non-device & $<2$ & 33.8 & 5 & 25 & 6 \\
\hline 4 & 21 & Device & $2-4$ & 30 & 7 & 28.8 & 7 \\
\hline 5 & 43 & Device & $2-4$ & 31.8 & 6 & 30 & 6 \\
\hline 6 & 44 & Device & $<2$ & 29 & 4 & 29 & 5 \\
\hline 7 & 30 & Device & $<2$ & 25 & 5 & 22.5 & 6 \\
\hline 8 & 14 & Device & $>4$ & 30 & 6 & 26 & 7 \\
\hline 9 & 19 & Non-device & $2-4$ & 38 & 5 & 35 & 5 \\
\hline 10 & 17 & Non-device & $<2$ & 25 & 7 & 25 & 7 \\
\hline Total (mean) & 30.8 & & & 30.36 & 5.7 & 28 & 6.1 \\
\hline
\end{tabular}

In this study, the only results of pediatric patients were evaluated and then a revision rate in children demonstrated as $6.7 \%$. The mean time between implantation/reimplantation was found as 30.8 (range, 1-71) months and it can be seen in Table 1.

According to European consensus guidelines [5], device failure rate in our pediatric CI patients was obtained as $4.69 \%$ and cumulative survival graphic of our pediatric patients was shown in Fig. 1.

Hard failures were $60 \%$ of revision surgeries (6/10) and soft failures were $10 \%(1 / 10)$. All of 6 hard failures of patients had a history of head trauma. Especially one of them (patient number 5) had undergone reimplantation 3.5 years after the first implantation. His device was cracked. He was very active and then consulted by psychiatry and had diagnosed as hyperactivity disorders and treated with medicine before the revision surgery. The other 5 children with the history of trauma were children from rural areas who helped their families in farming. One of the 2 children with hermetic failure detected gave a history of falling from the tractor; the other was learned through a psychologist that he was exposed to parental violence and was included in the child protection program. The other children with hard failure were found to have electrode breakage in in vivo integrity test and ex vivo test (Table 2).

One of our patients (patient number 1) had diagnosed as a soft failure of the device. He had complaint of progressive decrement of hearing and intermittent disruption of the function of the device. In the operating room, an in vivo integrity test was done, but the test was normal. Then CT was taken and reported as electrodes were all placed inside the cochlea. After an increase in his complaints, reimplantation was planned. After reimplantation, his complaints had gone away and he was satisfied.

One patient (patient number 9) was undergone revision surgery because of infection. He had otitis media with effusion

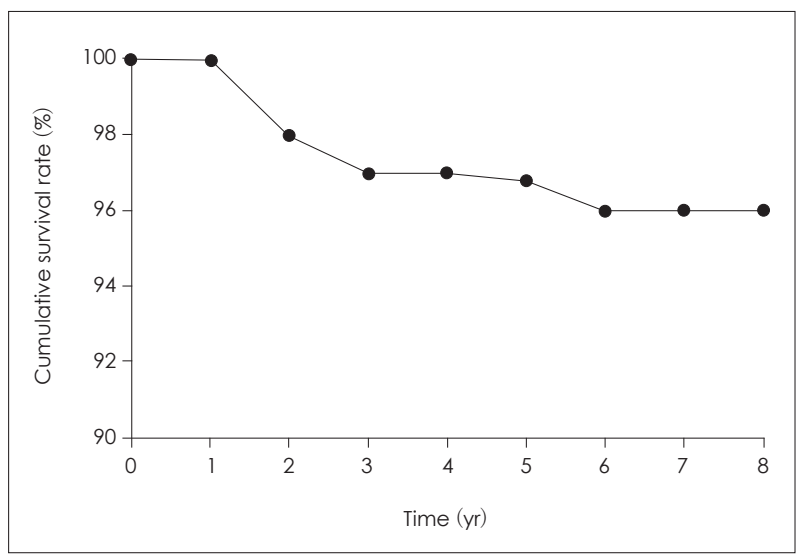

Fig. 1. Cumulative survival rate graphic for $\mathrm{Cl}$ devices appiled in our clinic.

before the first implantation and had undergone adenoidectomy and bilateral ventilation tube insertion one month before CI operation. However, 20 months later of implantation, infected drainage was started and continued despite local and systemic antibiotics. Then the implant did not work and he had undergone revision surgery.

Electrode migration to facial recess was seen in one case. In this patient (patient number 3), 8 electrodes of the implant were seen outside and encircled with soft tissue and fibrosis around the facial recess. Reimplantation with a new device and normal insertion was done after the cleaning of these tissue materials.

Patient with number 10 had been applied the first implantation at one year old and then other left side was implanted. But she had not heard from this side although working of the device was proven with in vivo tests. Then CT was taken and reported the left narrow internal auditory canal (IAC). The comprehensive analysis of revision reasons of patients was 
Table 2. Analysis of the reasons of cochlear implant revision operations in our clinic

\begin{tabular}{|c|c|c|c|c|}
\hline Patient no & Clinical/surgical opinion & Integrity test & Ex vivo test & Revision reason result \\
\hline 1 & Progressive decrement in audiological performance & Normal & - & Soft failure \\
\hline 2 & Head trauma & Abnormal & $\begin{array}{l}\text { Impact failure } \\
\text { (electrode breakage) }\end{array}$ & Hard failure \\
\hline 3 & Electrode array migration to facial recess & Normal & Medical reason & Electrode migration \\
\hline 4 & Head trauma & Abnormal & $\begin{array}{l}\text { Impact failure } \\
\text { (electrode breakage) }\end{array}$ & Hard failure \\
\hline 5 & Head trauma & Abnormal & Impact failure (cracked) & Hard failure \\
\hline 6 & Head trauma & Abnormal & Hermeticity failure & Hard failure \\
\hline 7 & Head trauma & Abnormal & $\begin{array}{l}\text { Impact failure } \\
\text { (electrode breakage) }\end{array}$ & Hard failure \\
\hline 8 & Head trauma & Abnormal & Hermeticity failure & Hard failure \\
\hline 9 & $\begin{array}{l}\text { EOM at preimplant period/ history of ventilation tube/ } \\
\text { infection post-operative period }\end{array}$ & Normal & Medical reason & Infection \\
\hline 10 & Poor outcome/narrow IAC & Normal & Normal & Poor outcome from left \\
\hline
\end{tabular}

EOM, effusion with otitis media; IAC, internal auditory canal

given in Table 2.

Auditory performances of the patient were assessed with free-field PTA threshold and CAP score. Speech discrimination scores were not given because the mean age was low and there were no results for the whole. All patients showed better or same post-reimplantation performance compared with prereimplantation results and the results were statistically insignificant. Audiological results were also summarized in Table 1.

\section{Discussion}

The most important result in this study is that the most common reason for revision in our clinic is head trauma, which can be prevented by the patient and his family. Our institute is located in the south of the country and serves a lower socioeconomic segment compared to other parts of the country. Syrian immigrant children also apply to our center for surgery. In the majority of our patient group, families have more than two children; the level of education is low. This was thought to be also influential in the history of head trauma. İndeed, in a study from India, Bhadania, et al. [13] found that 3 patients of 250 patients with CIs had hard failure and two of whom had a history of head trauma. Olgun, et al. [16] published their results of $946 \mathrm{CI}$ surgeries that undergone in an institute in the west of the country. There was no head injury in their patients and device failure rates were given as $3.8 \%$. This rate would have been the same if there were no head injuries in our series. We thought that in larger series, a comprehensive investigation is needed regarding the relationship between education and socioeconomic status of the family and CI failure.

During Oticon surgery, there is no need to make bone-bed for the recipient on the bony cortex because the recipient is fixed with two titanium screws. This shortens the surgical time, but in the early postoperative period, the recipient is located just under the skin, and it may create a sensitive situation for traumas. However, over the years, new bone formation envelops the recipient. We have seen this new bone and the spontaneous bone-bed formation around the recipient in all revision surgeries. When we look at the studies in the literature that give the results of revision surgery performed with more than one brand from our country, our revision rates are compatible. The most common major problem of revision in these studies is device failure but no differences were observed between the brands and there is no information about trauma history as a cause of failure $[17,18]$. The sensitivity to the trauma may be the disadvantage of our patients because of the surgical difference of the Oticon device. However, this idea can only be proved by giving the medical history, clinical findings, in vivo integrity test and ex vivo findings of patients who underwent revisions with more than one brand.

In this study, we have given only the results of a device we apply the most so that other brands do not affect the results. Our institute had signed a contract with Neurelec/Oticon between 2010 and 2018 and we were able to achieve all the results including in vivo and ex vivo test results during this time. In the literature, the failure rate and revision rate of this company have been presented in the limited publication. Our results actually do not reflect the real results of this manufacturer, as the hard failures due to the head trauma are high. However, when all these publications are considered, our study is the first study given the comprehensive analysis of revision surgeries of Oticon/Neurelec from our country. In our study, the overall explantation/reimplantation revision rate was 5.2\% and the device failure rate was $4.69 \%$. 
Table 3. Reimplantation of Neurelec/Oticon in the literature

\begin{tabular}{|c|c|c|c|c|}
\hline Study & $\begin{array}{c}\text { Digisonic }{ }^{\circledR} \text { implanted } \\
\text { patients }\end{array}$ & $\begin{array}{l}\text { All explantation/ } \\
\text { reimplantation patients }\end{array}$ & $\begin{array}{c}\text { Digisonic }{ }^{\circledR} \text { reimplanted } \\
\text { patients }\end{array}$ & Reason \\
\hline Battmer, et al. [19] & $527 \mathrm{Cl}$ of all patients & 488 patients & 17 patients & Device failure \\
\hline Guevara, et al. [21] & 166 patients & 5 explanted & 1 reimplanted & One head trauma \\
\hline Rădulescu, et al. [20] & 672 patients & 15 explantation/6 device failure & Not reported & One head trauma \\
\hline Farinetti, et al. [22] & 26 patients & 14 patients & Not reported & Not reported \\
\hline Sterkers, et al. [15] & 24 patients & 42 patients & 2 patients & Not reported \\
\hline Blanchard, et al. [10] & 36 patients & 50 patients & 1 patients & Menengitis \\
\hline
\end{tabular}

$\mathrm{Cl}, \mathrm{cochlear}$ implant

Battmer, et al. [19] gave the failure results of European CI centers in 2007 and found 17 failure of 527 device of Neurelec MXM (Laboratoires MXM, Vallauris, France) all around Europe. In that study, reason or age group were not reported. Rădulescu, et al. [20] showed the reliability of Oticon/Neurelec Digisonic $^{\circledR}$ (Option Medical/Neurelec SAS, Vallauris, France) with a multicenter independent study with a follow-up of five years in 2013 and showed that overall explantation rate was $2.23 \%$ and failure rate was $0.83 \%$ for children with Digisonic $^{\circledR}$. Other revision reports of CI centers were not comprehensive for the real analysis of failure rate of Oticon/Neurelec (Table 3) [10,21,22]. Our revision rates were higher than the multicenter study of failure of Oticon/Neurelec actually. However, head trauma was happened in the only one case in their series as distinct from our study.

With a follow-up period of eight years, our revision rate of children was $6.7 \%$. This is similar with the revision rate of other studies in literature [1,10,23]. Brown, et al. [24] found that the revision rate was higher in pediatric population with $7.3 \%$ than adult with $3.8 \%$ and also showed that the hard failure was more seen reason with the rate of $55 \%$ in their study. In our study, hard failure was also most seen as the cause of revision surgery. We found that all of 6 children with hard failure had a specific history of head trauma $(60 \%, 6 / 10)$. A child with head trauma was consulted with psychiatry because of extreme moving child and he was treated for hyperactivity disorder prior to revision surgery. The other child with head trauma was included in the child protection program because of their parents' violence. It is thought that especially children who develop hard failure due to head trauma should continue psychiatric evaluation which has begun before surgery [25].

It has been suggested that another reason of higher rate of revision among pediatric population is infection $[1,26]$. Sunde, et al. [14] found that infection was responsible of $0.9 \%$ of their revision surgery. Routine use of antibiotic irrigation of surgical site and aggressive management of otitis media with tympanostomy tube placement is important for prevention of this complication [24]. Although we do not wash the implant site with antibiotics, we do not have any surgical wound or flap complications. Actually, we thought that small postauricular incision and small tight subperiosteal pocket for receiver-stimulator is more important to prevent flap necrosis and surgical wound complication. In one patient, we performed aggressive treatment for otitis media with adenoidectomy and tympanostomy tube placement before CI, but we did not prevent middle ear infection. In this patient, tympanostomy tube placement was applied only one month before than CI procedure. In this regard, we suggested that if the candidate has a serious otitis media, tymponostomy tube placement is applied immediately and then surgeon should wait and be sure about healing of middle ear mucosa. If necessary, mastoidectomy may be done before the planning CI.

Electrode array migration is less common cause of revision surgery. Olgun, et al. [16] found 6 cases with misdirection of electrode array in their analysis of 957 cases and only one had needed revision surgery. Brown, et al. [24] had found the electrode migration in his cases with history of meningitis. Idiopathic fibrosis and ossification of the basal turn in these cases may cause extrusion of electrode. In our patients, one case had electrode migration. He had not history of meningitis and revision surgery had performed with ease.

The initial diagnosis may rarely be incompatible with the diagnosis from the company in the patient who was decided to undergo revision surgery. Distinguin, et al. [26] suggested a decision-tree for diagnosis and misclassified the diagnosis of only nine cases in their 64 patients. They claimed that the history of trauma may cause misclassification and may mislead the surgeon. In our study, clinical, surgical and in vivo integrity test results were compared with ex vivo analyses of company in all patients. However, head trauma has actually caused device breakage or silicone tears in our cases and has not cause misdiagnosis. In brief speaking, the diagnosis of soft failure in our cases is more difficult actually. It is a very difficult challenge to reveal the soft failure problem without doing reimplantation surgery. Because, the patients with soft failure have non-specific and unproven complaints such as pain, tinnitus or intermittent interruption of signals. In this situation, all of 
in vivo and ex vivo tests are normal and the only thing that shows that the problem is in the device, replacing the device can correct all complaints [6,27]. In our two cases (patient 1 and 10), we thought soft failure before the revision surgery. Patient 1 benefited from device replacement. However patient 10 did not benefit from revision and diagnosed as narrow IAC. We investigated all CT images of this child again and realize that the narrow IAC was present on the left side. Sennaroglu [28] mentioned in his publication about poor outcome of narrow IAC because of a possibility of absent or hypoplastic cochlear nerve. Actually, if the surgeon recommend to the patient for revising surgery of CI, he should investigate again the $\mathrm{CT}$ and MRI images with detail and inform the patient and family about possibility of doubtful result of reimplantation.

Audiological outcomes of CI revision surgery has been investigated in many study with different tests $[1,7,11,15]$. Manrique-Huarte, et al. [7] tested audiological outcomes with PTA and speech audiometry and they claimed that reimplantation due to device failure did not record significance changes in speech perception before and after reimplantation. Sterkers, et al. [15] used open-sentence testing in quiet and in noise together with speech perception test scores. They found that reimplantation has no negative effect on auditory outcome and suggested that in rare cases; speech perception outcome may not improve, requiring a specific rehabilitation program. Yeung, et al. [1] and Reis, et al. [11] also showed similar results that reimplantation cause same or better SRS than the last measured score before the surgery. We compared PTA thresholds $(0.5$, 1,2 , and $4 \mathrm{kHz}$ ) and CAP scores of preoperative last measured results and the postoperative results. We had got light better or same results on postoperative records. CAP is a classification defined by Archbold, et al. [12] in 1995 and revised in 2010. The CAP score is an index describing the highest typical level of functional hearing in everyday life. This scale can provide brief information about speech and conversation performance of CI patients [29]. Bhadania, et al. [13] used CAP scale to interpret their results of CI revision surgery and found good performance in postoperative period. Actually, we thought that some protocols and international scales are necessary to evaluate the auidological outcomes of CI revision surgery.

This study has some limitations. Retrospective and single company results have been given and it has not unfortunately huge series and long-term follow-up. However, our study is the unique study that gives only the revision rate of the Oticon/ Neurelec brand and gives very valuable audiological and surgical information about this brand during and after revision surgery. Our clinic is one of the centers in Europe that used high number of Oticon brand. There is only one study about the results of Oticon/Neurelec Digisonic ${ }^{\circledR}$ brand in the literature and it is a multi-center study. However, there has been no study that gives the result of a single-center, single-brand revision of this brand besides our study especially after the merger with Oticon and Neurelec.

If studies with surgical results on this brand increase, the company may provide its own technological development. The bottom of the receiver of this brand is not closed like other brands. The electrode and/or wires coming out from the receiver are easily visible through a thin hermetic silicone coating. This silicone coated receiver has two screw holes at the end. If the bone bed is opened, this screwing cannot be done and the electrodes and/or wires can be bent. For this reason, it may be beneficial to cover the bottom of receiver with a stronger material instead of opening a bone bed in surgery. This could provide a protection against the trauma which is the most important cause of CI device failure in children, especially in developing countries as shown in this study.

In order to reduce revisions due to trauma, not only the selfdevelopment of the brand, but also the socioeconomic and educational level of the country should be improved. We should educate not only the family but also the child about end results of trauma and the protection himself. Before the implantation, the family should be informed about the device failure probability and should understand that in this situation, the child's education may be delayed. Creating a safe home and in-school environment is very important. Surgeon and audiologist should be alert and doubtful for hyperactivity disorders.

In conclusion, cochlear reimplantation is a safe and welltolerated surgery with good performance in postoperative audiological results. Revision surgery protocols regarding surgical method and postoperative audiological assessment should be standardized with publishing results of other CI center. These protocols could help the companies to develop their technology as well as the surgeons to manage the revision cases. It is very important to provide a safe school and home environment and to keep family education at a high level for reducing reimplantation in children due to the trauma. Especially for active child or those who have experienced severe trauma preoperative psychiatric consultation should be continued postoperatively.

\section{Acknowledgments}

This study was supported by Baskent Univercity Research Fund.

\section{Conflicts of interest}

The authors have no financial conflicts of interest.

\section{Author Contributions}

Conceptualization: all authors. Data curation: Fulya Ozer, Haluk Yavuz, Ismail Yilmaz. Formal analysis: Ismail Yilmaz. Funding ac- 
quisition: Levent N. Ozluoglu. Investigation: Fulya Ozer, Haluk Yavuz. Methodology: Fulya Ozer, Haluk Yavuz, Ismail Yilmaz. Project administration: Fulya Ozer. Resources: Fulya Ozer, Haluk Yavuz. Software: Fulya Ozer, Ismail Yilmaz. Supervision: Levent N. Ozluoglu. Validation: Fulya Ozer, Levent N. Ozluoglu. Visualization: Haluk Yavuz, Ismail Yilmaz. Writing — original draft: Fulya Ozer, Haluk Yavuz. Writing - review \& editing: Fulya Ozer, Levent N. Ozluoglu. Approval of final manuscript: all authors.

\section{ORCID iDs}

\section{Fulya Ozer}

Haluk Yavuz

Ismail Yilmaz

Levent N. Ozluoglu

\section{REFERENCES}

1) Yeung J, Griffin A, Newton S, Kenna M, Licameli GR. Revision cochlear implant surgery in children: surgical and audiological outcomes. Laryngoscope 2018;128:2619-24.

2) Amaral MSAD, Reis ACMB, Massuda ET, Hyppolito MA. Cochlear implant revision surgeries in children. Braz J Otorhinolaryngol 2019;85:290-6.

3) Hochmair-Desoyer I, Burian K. Reimplantation of a molded scala tympani electrode: impact on psychophysical and speech discrimination abilities. Ann Otol Rhinol Laryngol 1985;94:65-70.

4) Zeitler DM, Budenz CL, Roland JT Jr. Revision cochlear implantation. Curr Opin Otolaryngol Head Neck Surg 2009;17:334-8.

5) European consensus statement on cochlear implant failures and explantations. Otol Neurotol 2005;26:1097-9.

6) Balkany TJ, Hodges AV, Buchman CA, Luxford WM, Pillsbury CH, Roland PS, et al. Cochlear implant soft failures consensus development conference statement. Otol Neurotol 2005;26:815-8.

7) Manrique-Huarte R, Huarte A, Manrique MJ. Surgical findings and auditory performance after cochlear implant revision surgery. Eur Arch Otorhinolaryngol 2016;273:621-9.

8) Mishu C, Klodd DA, Redleaf M. Cochlear implant electrode array exposure: a delayed complication. Ear Nose Throat J 2017;96:E40-3.

9) Wang JT, Wang AY, Psarros C, Da Cruz M. Rates of revision and device failure in cochlear implant surgery: a 30-year experience. Laryngoscope 2014;124:2393-9.

10) Blanchard M, Thierry B, Glynn F, De Lamaze A, Garabédian EN, Loundon N. Cochlear implant failure and revision surgery in pediatric population. Ann Otol Rhinol Laryngol 2015;124:227-31.

11) Reis M, Boisvert I, Looi V, da Cruz M. Speech recognition outcomes after cochlear reimplantation surgery. Trends Hear 2017;21:1-9.

12) Archbold S, Lutman ME, Marshall DH. Categories of auditory performance. Ann Otol Rhinol Laryngol Suppl 1995;166:312-4.

13) Bhadania SR, Vishwakarma R, Keshri A. Cochlear implant device failure in the postoperative period: an institutional analysis. Asian $\mathrm{J}$ Neurosurg 2018;13:1066-70.

14) Sunde J, Webb JB, Moore PC, Gluth MB, Dornhoffer JL. Cochlear implant failure, revision, and reimplantation. Otol Neurotol 2013; 34:1670-4.

15) Sterkers F, Merklen F, Piron JP, Vieu A, Venail F, Uziel A, et al. Outcomes after cochlear reimplantation in children. Int J Pediatr Otorhinolaryngol 2015;79:840-3.

16) Olgun Y, Bayrak AF, Catli T, Ceylan ME, Aydin R, Duzenli U, et al. Pediatric cochlear implant revision surgery and reimplantation: an analysis of 957 cases. Int J Pediatr Otorhinolaryngol 2014;78:1642-7.

17) Batuk MO, Cinar BC, Yarali M, Bajin MD, Sennaroglu G, Sennaroglu L. Twenty years of experience in revision cochlear implant surgery: signs that indicate the need for revision surgery to audiologists. J Laryngol Otol 2019;133:903-7.

18) Dağkıran M, Tarkan Ö, Sürmelioğlu Ö, Özdemir S, Onan E, Tuncer $\ddot{U}$, et al. Management of complications in 1452 pediatric and adult cochlear implantations. Turk Arch Otorhinolaryngol 2020;58:16-23.

19) Battmer RD, O'Donoghue GM, Lenarz T. A multicenter study of device failure in European cochlear implant centers. Ear Hear 2007;28: 95S-9.

20) Rădulescu L, Cozma S, Niemczyk C, Guevara N, Gahide I, Economides J, et al. Multicenter evaluation of Neurelec Digisonic $₫$ SP cochlear implant reliability. Eur Arch Otorhinolaryngol 2013;270:150712.

21) Guevara N, Sterkers O, Bébéar JP, Meller R, Magnan J, Mosnier I, et al. Multicenter evaluation of the digisonic SP cochlear implant fixation system with titanium screws in 156 patients. Ann Otol Rhinol Laryngol 2010;119:501-5.

22) Farinetti A, Ben Gharbia D, Mancini J, Roman S, Nicollas R, Triglia JM. Cochlear implant complications in 403 patients: comparative study of adults and children and review of the literature. Eur Ann Otorhinolaryngol Head Neck Dis 2014;131:177-82.

23) Lane C, Zimmerman K, Agrawal S, Parnes L. Cochlear implant failures and reimplantation: a 30-year analysis and literature review. Laryngoscope 2020;130:782-9.

24) Brown KD, Connell SS, Balkany TJ, Eshraghi AE, Telischi FF, Angeli SA. Incidence and indications for revision cochlear implant surgery in adults and children. Laryngoscope 2009;119:152-7.

25) Grigorian A, Nahmias J, Dolich M, Barrios C Jr, Schubl SD, Sheehan $\mathrm{B}$, et al. Increased risk of head injury in pediatric patients with attention deficit hyperactivity disorder. J Child Adolesc Psychiatr Nurs 2019;32:171-6.

26) Distinguin L, Blanchard M, Rouillon I, Parodi M, Loundon N. Pediatric cochlear reimplantation: decision-tree efficacy. Eur Ann Otorhinolaryngol Head Neck Dis 2018;135:243-7.

27) Celerier C, Rouillon I, Blanchard M, Parodi M, Denoyelle F, Loundon N. Pain after cochlear implantation: an unusual complication? Otol Neurotol 2017;38:956-61.

28) Sennaroglu L. Cochlear implantation in inner ear malformations--a review article. Cochlear Implants Int 2010;11:4-41.

29) Vashist S, Singh S. CSF gusher in cochlear implant surgery-does it affect surgical outcomes? Eur Ann Otorhinolaryngol Head Neck Dis 2016;133 Suppl 1:S21-4. 